\title{
The Efficacy of Ostrich Antibodies to Dihydrotestosterone and $5 \alpha$-Reductase in the Restoration of Hair Growth
}

\author{
Yasuhiro Tsukamoto1,2, Barry Hendler ${ }^{3}$, Stuart Greenberg ${ }^{*}{ }^{(}$, , Carol Epstein $^{2}$ \\ ${ }^{1}$ Department of Animal Sciences, Graduate School of Environmental \& Biological Sciences, Kyoto Prefecture University, Kyoto, Japan \\ ${ }^{2}$ Clinical Studies, Ostrich Pharma USA, West Newton, Massachusetts, USA \\ ${ }^{3}$ Microfollicular Hair Transplantation, Ambler, Pennsylvania, USA \\ Email: ^sgreenberg@ostrigen.com
}

How to cite this paper: Tsukamoto, Y., Hendler, B., Greenberg, S. and Epstein, C. (2019) The Efficacy of Ostrich Antibodies to Dihydrotestosterone and $5 \alpha$-Reductase in the Restoration of Hair Growth. Health, 11, 1320-1330.

https://doi.org/10.4236/health.2019.1110101

Received: September 14, 2019

Accepted: October 13, 2019

Published: October 16, 2019

Copyright $\odot 2019$ by author(s) and Scientific Research Publishing Inc. This work is licensed under the Creative Commons Attribution International License (CC BY 4.0).

http://creativecommons.org/licenses/by/4.0/

\begin{abstract}
The hormone dihydro testosterone (DHT) and the catalyst $5 \alpha$-reductase are key contributors to hair loss due to androgenetic alopecia (AGA). Also, dermal bacteria and their toxins have been implicated in hair loss. A preliminary study by the authors with six male volunteer subjects showed that ostrich antibodies against above causative substances were quite effective in promoting hair regrowth. The present study included 13 men and 4 women, with ages ranging from 39 to 78. Pre- and post-treatment hair counts were also incorporated into the study procedure. In all instances but one, the subjects had a hair-growth effect about 3 months after the antibody was applied to the scalp, and the study participants with hair growth were pleased with the results and with the ease of use of the antibody solution.
\end{abstract}

\section{Keywords}

Alopecia, AGA, Ostrich, Antibody

\section{Introduction}

Dihydrotestosterone (DHT) and $5 \alpha$-reductase have been shown to be involved in the male pattern baldness of androgenic alopecia (AGA) [1] [2] [3]. It has been shown that reducing the production of DHT promotes hair growth in AGA [4]. Topically applied minoxidil and orally administered finasteride have been shown to be effective in countering the effects of DHT and reducing the production of DHT, respectively [5] [6] [7]. It has also been suggested that abnormal propagation of pathogens such as dermal bacteria and their toxins induce hair loss [8]. 
We have so far developed a method to promote hair growth by specifically suppressing hair loss-inducing factors using antibodies. Antibodies are immunoglobulins produced by $\beta$-cells when a foreign substance such as pathogens enters the body. The antibodies function to detoxify or eliminate the antigens. Antibodies can also be effective externally. The characteristic "antigen-antibody" reaction usually targets only the inducing antigen. Usually, therapeutic antibodies are produced in mice, rabbits or cultured cells, and their production costs are very high.

As discussed in a previous article by the authors [9], birds produce an antibody form, immunoglobulin Y ("IgY"), which is passed into the yolk of their eggs [10]-[15]. The antibodies have been purified from egg yolk and applied to a wide range of pathogen targets, such as Pseudomonas aeruginosa, Staphylococcus aureus, and Vibrio cholerae [15] [16].

The ostrich immune system is an effective producer of IgY [16]-[23]. IgY is produced within two weeks after vaccination with an antigen. Ostrich IgY is less sensitive to heat and more resistant to acid than the IgY from other avian species. The molecular weight of ostrich IgY is higher than that of mammalian IgG, since the Fc fraction of ostrich IgY is heavier. Ostriches produce IgY in large volume at comparatively low cost. About 4 grams of IgY are in each egg yolk, and an ostrich hen can produce as many as 100 eggs per year over a 55-year lifetime. Commercial exploitation of ostrich antibodies has begun. For example, ostrich IgY against dermal bacteria has been added to skin cosmetic products [20]. Accordingly, ostrich IgY against DHT, $5 \alpha$-reductase, and the dermal bacteria form a promising cocktail of antibodies to stimulate hair growth in cases of AGA.

\section{Production of Antibodies against DHT, $5 \alpha$-Reductase and Dermal Bacteria}

The vaccination of ostriches and subsequent purification of IgY against DHT and $5 \alpha$-reductase from the yolks of their eggs is described in the authors' preceding study [9]. As described in [23], IgY is purified via a proprietary process from the yolk of eggs from hens vaccinated with homogenates of skin bacteria, specifically $S$. aureus and $P$. aeruginosa. Solutions of IgY against DHT, $5 \alpha$-reductase, $S$. aureus, and $P$. aeruginosa were combined to form the active material to be mixed with base material for testing.

\section{Materials and Methods}

\subsection{Results of a Preliminary Study}

A study was performed with six male subjects, and all subjects achieved hair growth [9]. However, the antibodies were mixed into Exoderm ${ }^{\circ}$, a commercial hair conditioner from Bentlin Products LLC, and the subjects did not feel it was particularly easy or convenient to use in the protocol of the study. Another shortcoming of the preliminary study was that the results were qualitatively evaluated via before and after photographs, but there were no quantitative measures of growth. 


\subsection{Description of Current Study}

To overcome the limitations of the preliminary study, a larger study was performed. In this study male and female subjects used material based on a solution of ethyl alcohol in distilled water (20\% alcohol). The study procedure included before and after hair counts to establish the basis for quantitative evaluation.

The objectives of the study were 1) to make a qualitative visual assessment of hair growth and 2) to measure actual hair count change over a three-month period.

Seventeen individuals were recruited from among his patients and others by author Dr. Hendler to participate in the study. Brief information on these subjects is presented in Table 1. That information covers age, gender, degree of baldness (Norwood Scale ${ }^{1}$ for males and Ludwig Scale ${ }^{2}$ for females), and the number of hair transplantations performed, if any.

The subjects were asked to use the material daily for a period of three months. They were instructed to leave the material on at least ten minutes before washing their hair, but there was no requirement to wash their hair after application.

The study was performed at the medical office of Dr. Hendler. At the start of the study, photos were taken. Also, one square centimeter test areas along the midsagittal plane was marked and the location was recorded. A hair count was performed on the test area. Photos were taken and hair count was repeated at the end of three months.

Table 1. Brief information on the study subjects.

\begin{tabular}{|c|c|c|c|c|}
\hline Subject & Gender & Age (years) & Extent of Baldness & Prior Treatment \\
\hline 1 & $\mathrm{~F}$ & 76 & Ludwig Type II & None \\
\hline 2 & M & 48 & Norwood Type III Vertex & 2 Hair Transplantations \\
\hline 3 & $\mathrm{~F}$ & 75 & Ludwig Type I & None \\
\hline 4 & M & 76 & Norwood Type III Vertex & None \\
\hline 5 & M & 73 & Norwood Type V & 3 Hair Transplantations \\
\hline 6 & M & 71 & Norwood Type IV & None \\
\hline 7 & M & 76 & Norwood Type III Vertex & None \\
\hline 8 & M & 68 & Norwood Type 5A & None \\
\hline 9 & M & 70 & Norwood Type VII & 2 Hair Transplantations \\
\hline 10 & M & 60 & Norwood Type VI & 4 Hair Transplantations \\
\hline 11 & M & 75 & Norwood Type VI & 4 Hair Transplantations \\
\hline 12 & M & 39 & Norwood Type III Vertex & None \\
\hline 13 & M & 61 & Norwood Type VI & 3 Hair Transplantations \\
\hline 14 & M & 61 & Norwood Type VI & 3 Hair Transplantations \\
\hline 15 & M & 75 & Norwood Type V & 3 Hair Transplantations \\
\hline 16 & $\mathrm{~F}$ & 50 & Ludwig Type II & None \\
\hline 17 & $\mathrm{~F}$ & 78 & Ludwig Type II & 2 Hair Transplantations \\
\hline
\end{tabular}

${ }^{1}$ https://www.americanhairloss.org/men_hair_loss/the_norwood_scale.html

${ }^{2}$ http://worldhairinstitute.com/ludwig.shtml 


\section{Results}

Except for Subject 16, all subjects demonstrated hair regrowth. Subject 16 does not have AGA, but instead suffers from Central Centrifugal Cicatricial Alopecia (CCCA), a common cause of hair loss in African American women.

Photos of the subjects are presented in Figures 1-17 below.

Pre- and post-treatment hair counts per test area were also incorporated into the study procedure: in all instances but one, the subjects had a hair-growth effect about 3 months after the antibody was applied to the scalp of women (Figure 18) and men (Figure 19).

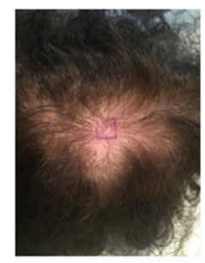

Pre: Mid-scalp

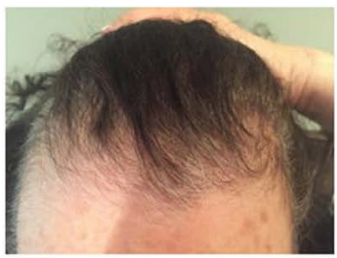

Pre: Hairline/Frontal

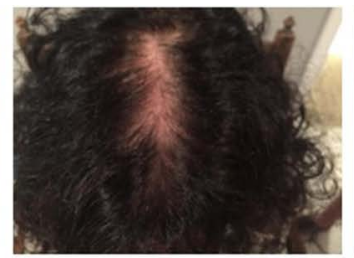

Post: Mid-scalp

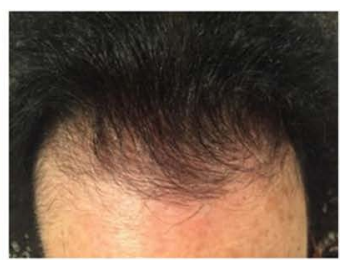

Post: Hairline/Frontal

Figure 1. Subject 1: female, age 76, Ludwig type II with no previous treatment (pre hair count 74; post hair count 112).

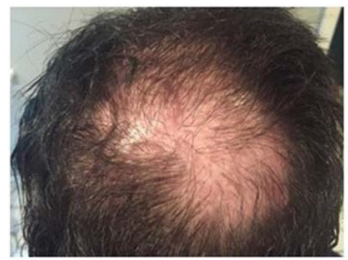

Pre: Vertex

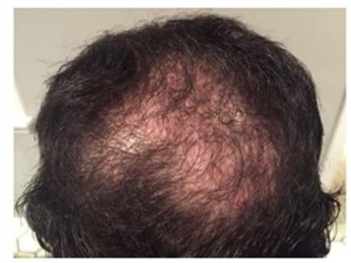

Post: Vertex

Figure 2. Subject 2: male age 48, Norwood type III vertex with two previous hair transplantations (pre hair count 22, post hair count 30 ).

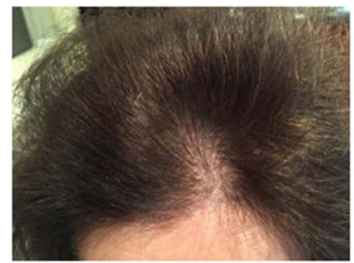

Pre: Hairline

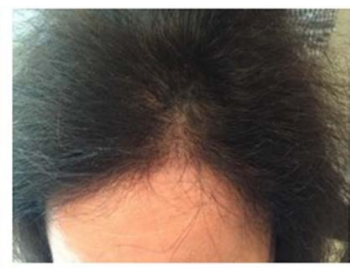

Post: Hairline

Figure 3. Subject 3: female age 76, Ludwig type I with no previous treatment (pre hair count 49 , post hair count 114 ); used test material every other day.

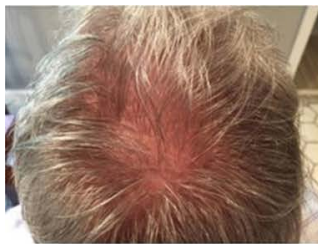

Pre: Vertex

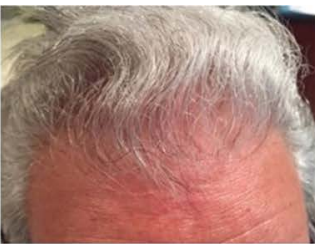

Pre: Hairline/Frontal

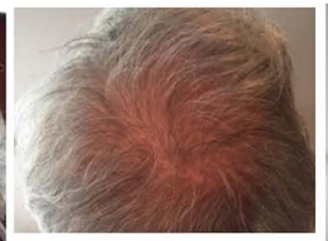

Post: Vertex

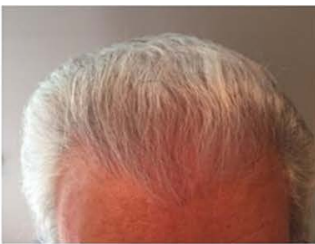

Post: Hairline/Frontal

Figure 4. Subject 4: male age 76, Norwood type III vertex with no previous treatment (two sample areas for hair counts: area 1 pre hair count 55 and post hair count 72; area 2 pre hair count 93 and post hair count 88 ). 


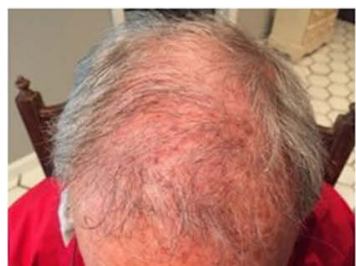

Pre: Frontal/Mid-scalp

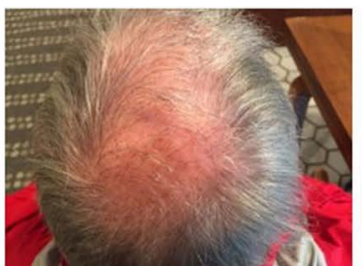

Pre: Vertex

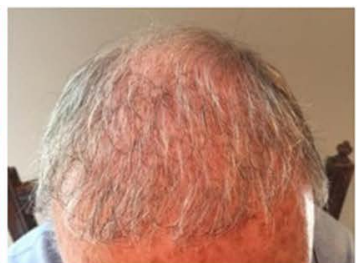

Post: Frontal/Mid-scalp

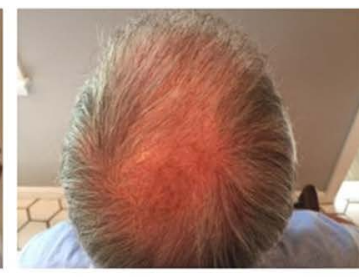

Post: Vertex

Figure 5. Subject 5: male age 73, Norwood type V with three precious hair transplantations (pre hair count 19 and post hair count 35 ).

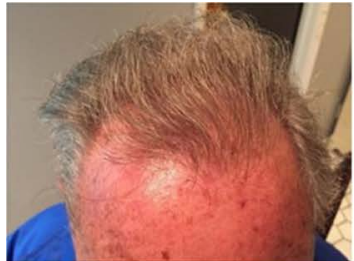

Pre: Frontal

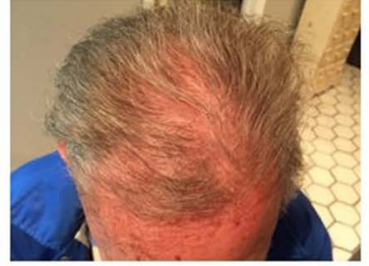

Pre: Mid-scalp

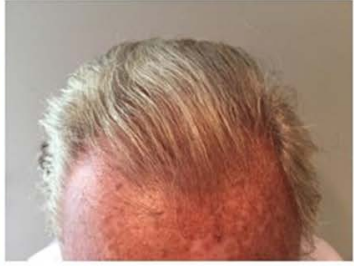

Post: Frontal

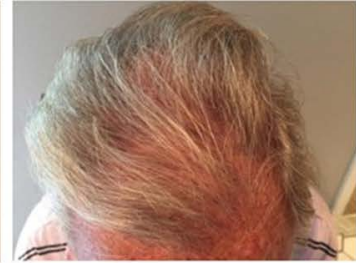

Post: Mid-scalp

Figure 6. Subject 6: male age 71, Norwood type IV with no previous treatment (pre hair count 53 and post hair count 82).

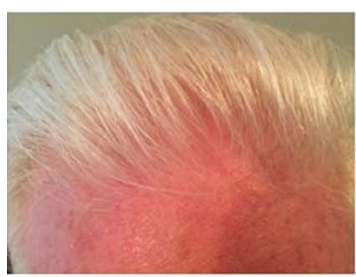

Pre: Hairline

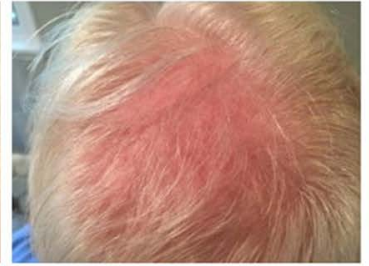

Pre: Vertex

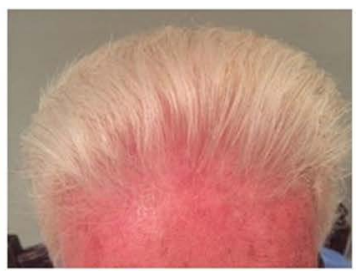

Post: Hairline

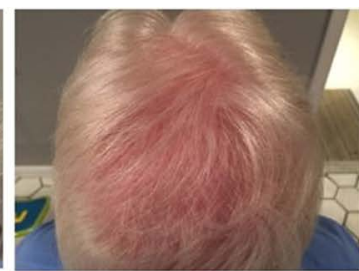

Post: Vertex

Figure 7. Subject 7: male age 76, Norwood type III vertex with no previous treatment (two sample areas for hair counts: area 1 pre hair count 28 and post hair count 46; area 2 pre hair count 22 and post hair count 63 ).

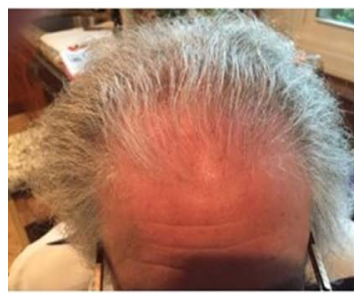

Pre: Frontal

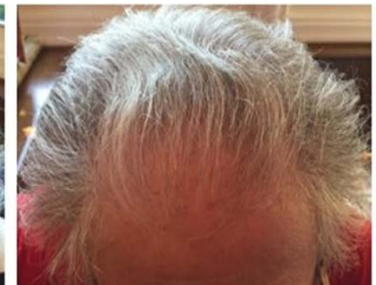

Post: Frontal

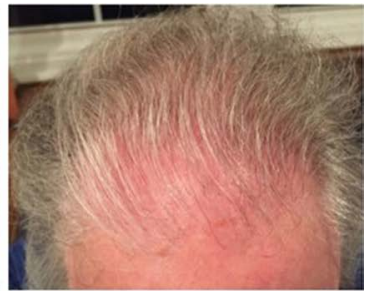

Post: Frontal

Figure 8. Subject 8: male age 68, Norwodd type 5A, no previous treatment (no hair count).

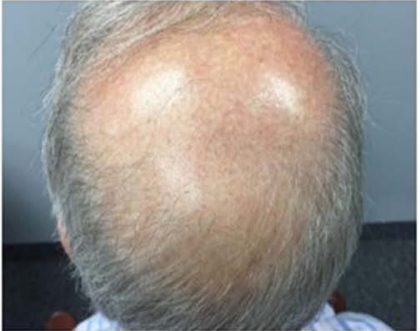

Pre: Vertex

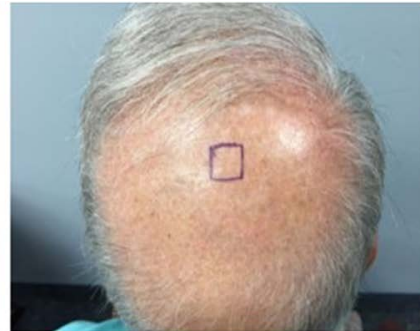

Post: Vertex

Figure 9. Subject 9: male age 70, Norwood type VII, two hair transplantations (pre hair count 1 and post hair count 5). 


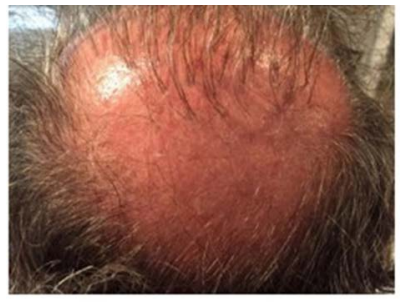

Pre: Vertex

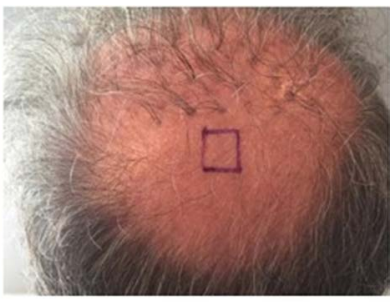

Post: Vertex

Figure 10. Subject 10: Male age 60, Norwood type VI, four hair transplantations (pre hair count 3 and post hair count 9).

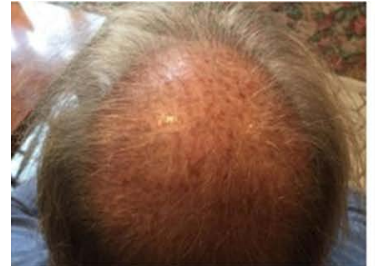

Pre: Vertex

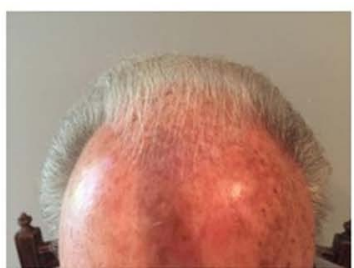

Pre:Hairline/Frontal

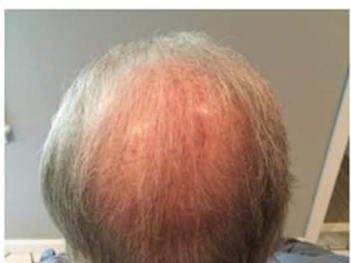

Post: Vertex

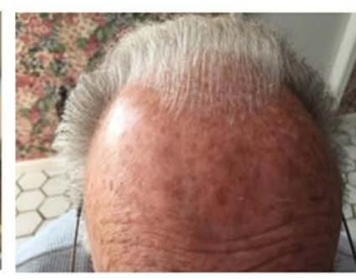

Post: Hairline/Frontal

Figure 11. Subject 11: male age 75, Norwood type VI, four hair transplanatations (no hair count).

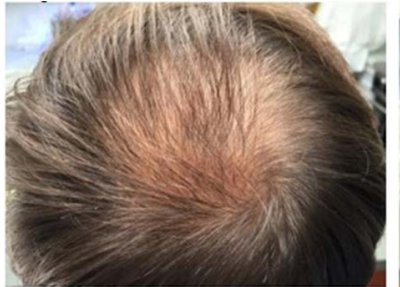

Pre: Vertex

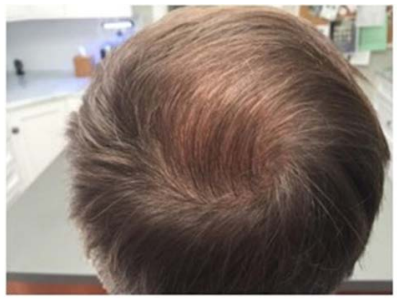

Post: Vertex

Figure 12. Subject 12: male age 39, Norwood type III vertex, no previous treatment (no hair count).

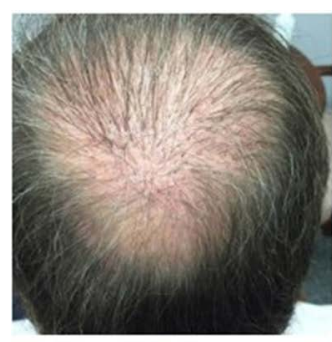

Pre: Vertex

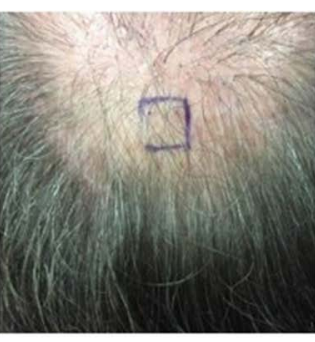

Pre: HC Site

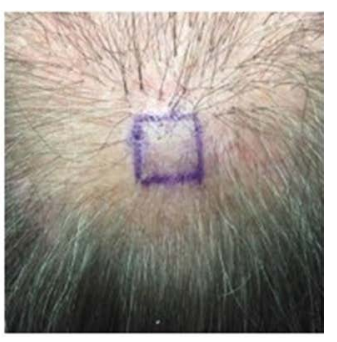

Post: HC Site

Figure 13. Subject 13: male age 61, Norwood type VI, three hair transplantations (pre hair count 15 and post hair count 33).

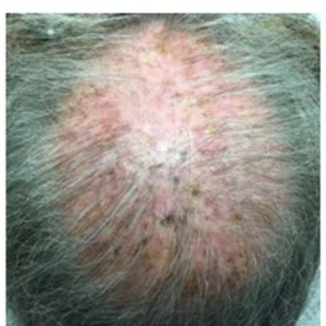

Pre: Vertex

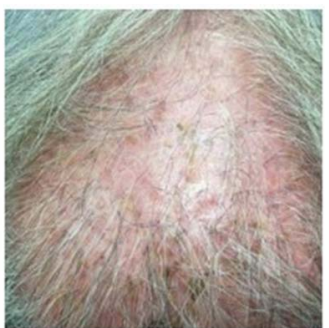

Post: Vertex

Figure 14. Subject 14: male age 61, Norwood type VI, three hair transplantations (no hair count). 


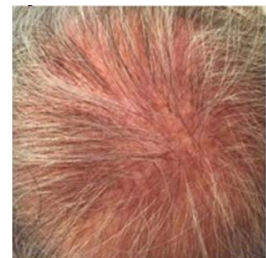

Pre: Vertex

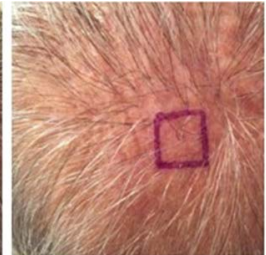

Pre: HC Site

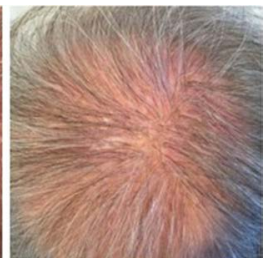

Post: Vertex

Figure 15. Subject 15: male age 75, Norwood type V, three hair transplantations (pre hair count 21 and post hair count 27).

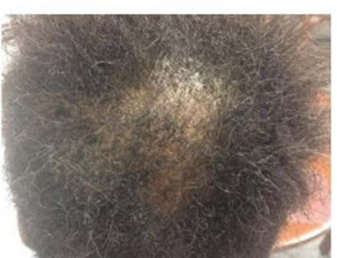

Pre: Vertex

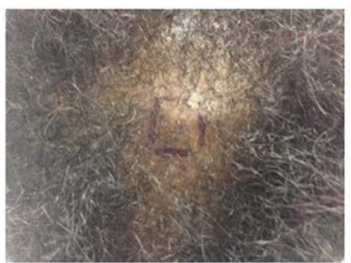

Pre: HC Site

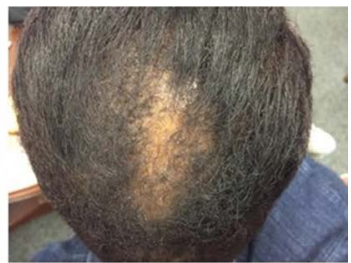

Post: Vertex

Figure 16. Subject 16: female age 50, CCCA and not AGA, no previous treatment (pre hair count 22 and post hair count 16).

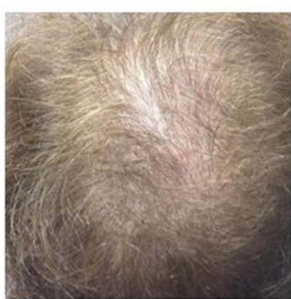

Pre: Mid Scalp

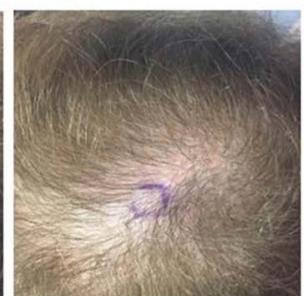

Pre: HC Site

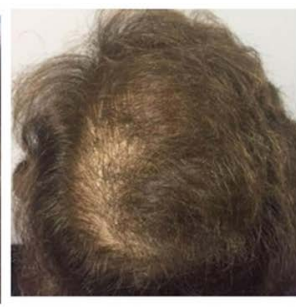

Post: Mid Scalp

Figure 17. Subject 17: female age 78, Ludwig type II, two previous hair transplantations (pre hair count 47 and post hair count 68).

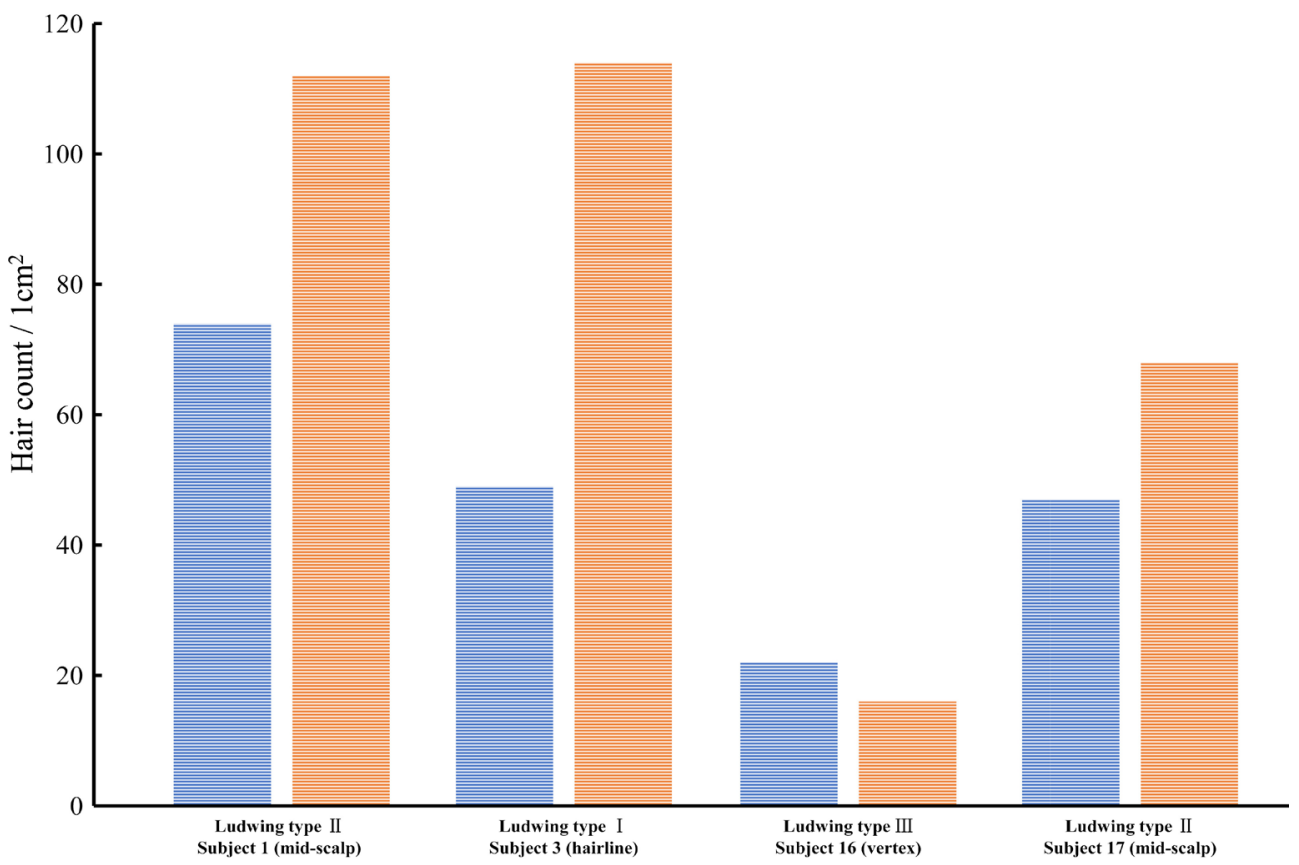

Figure 18. Graph of pre and post hair count for female participants. 


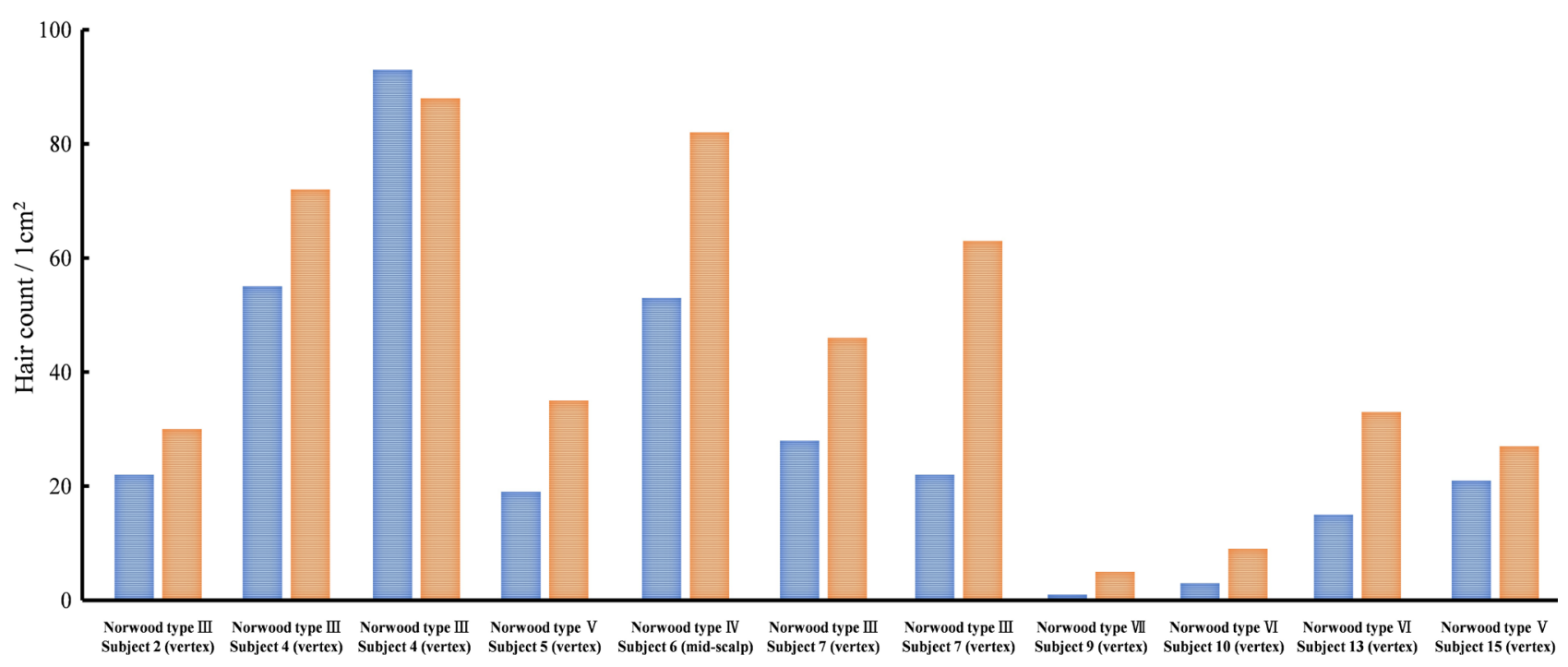

Figure 19. Graph of pre and post hair count for male participants.

Table 2. Effects on hair counts.

\begin{tabular}{cc}
\hline Average & Increase (\%) \\
\hline Overall Hair count & 71 \\
Male hair count & 69 \\
Female hair count & 73 \\
\hline
\end{tabular}

\subsection{Quantitative Results}

Pre and post hair counts were performed with 12 of the subjects with AGA. The following effects were observed (Table 2).

\subsection{Qualitative Feedback from Study Subjects}

All subjects found the new material extremely easy to use, which likely increased compliance with the requested usage protocol.

\section{Discussion}

In the present study, we have further demonstrated the effectiveness of ostrich antibodies to neutralization of DHT, $5 \alpha$-reductase, and two dermal bacteria in re-growing hair in the case of AGA.

Unlike the case with the oil-based material used in the preliminary study, the alcohol-based material proved to be convenient and easy-to-use. In experiments using mice, the immunofluorescent assay showed that ostrich antibodies immerse deep into the hair roots by using a base with alcohol (data not shown). It was suggested that the ostrich antibody efficiently binds to the hair loss factors in dermal tissues and neutralizes them, resulting in a hair growth effect.

Because of the limited number of subjects in the study, we realize the limitations in drawing conclusions that are too strong. However, we are more confident of the following observations and hypotheses: 
- Neutralization by ostrich antibodies of DHT, $5 \alpha$-reductase, $S$. aureus, and $P$. aeruginosa in hair follicles has a uniformly positive effect in restoring the growth of hair in cases of AGA;

- The ostrich antibodies in a 20 percent ethyl alcohol base is extremely easy and convenient to use;

- Mechanism is explained through the combination of three known effects: 1) vasoconstriction at the follicle base prevents hair stem cells in the follicle from initiating hair growth; 2) DHT in the follicle causes the vasoconstriction; and 3) ostrich antibodies to DHT, along with the enzyme $5 \alpha$-reductase that stimulates production of the DHT, have been demonstrated to neutralize DHT and $5 \alpha$-reductase so as to prevent vasoconstriction.

Based on comparison with results in the published literature, we can also draw limited conclusions that the ostrich antibody approach is significantly more effective than use of minoxidil and finasteride. For example, a comparable study [6] of $5 \%$ and $2 \%$ minoxidil solutions resulted in hair count increases of approximately $30 \%$, as compared with the $71 \%$ in this admittedly limited study.

To draw stronger conclusions, we are planning a larger study (40 to 50 subjects). The average subject age in the current study was 67 . It is believed that extended periods of balding reduce the viability of hair stem cells in follicles, so there can be no effective stimulation of hair growth in those follicles. It is anticipated that subjects at an earlier stage of hair loss will have even better results than those in this study. Accordingly, an age range of 25 to 60 will be targeted.

The next study should extend to six months, with photographs and hair count measurements taken at both three months and six months. The amount of the test article applied will also be calculated to correlate usage with Norwood and Ludwig scale designations. Furthermore, we plan to confirm the usefulness of ostrich antibodies for hair growth by conducting a double-blind test using only the base without antibody.

\section{Acknowledgements}

We thank Dr. Kazuhide Adachi, and Miss Saaya Ueno at Kyoto Prefecture University for their technical supports.

\section{Conflicts of Interest}

The authors declare no conflicts of interest regarding the publication of this paper.

\section{References}

[1] Sawaya, M.E. and Price, V.H. (1997) Different Levels of 5 Alpha-Reductase Type I and II, Aromatase, and Androgen Receptor in Hair Follicles of Women and Men with Androgenetic Alopecia. Journal of Investigative Dermatology, 109, 296-300. https://doi.org/10.1111/1523-1747.ep12335779

[2] Schweikert, H.U. and Wilson, J.D. (1974) Regulation of Human Hair Growth by Steroid Hormones. I. Testerone Metabolism in Isolated Hairs. The Journal of Clinical 
Endocrinology \& Metabolism, 38, 811-819. https://doi.org/10.1210/jcem-38-5-811

[3] Dallob, A.L., Sadick, N.S., Unger, W., et al. (1994) The Effect of Finasteride, a 5 AlphaReductase Inhibitor, on Scalp Skin Testosterone and Dihydrotestosterone Concentrations in Patients with Male Pattern Baldness. The Journal of Clinical Endocrinology \& Metabolism, 79, 703-706. https://doi.org/10.1210/jc.79.3.703

[4] Kaufman, K.D., Olsen, E.A., Whiting, D., et al. (1998) Finasteride in the Treatment of Men with Androgenetic Alopecia. Finasteride Male Pattern Hair Loss Study Group. Journal of the American Academy of Dermatology, 39, 578-589. https://doi.org/10.1016/S0190-9622(98)70007-6

[5] Lucky, A.W., Piacquadio, D.J., Ditre, C.M., Dunlap, F., Kantor, I., Pandya, A., Savin, R.C. and Tharp, M.D. (2004) A Randomized, Placebo-Controlled Trial of 5\% and 2\% Topical Minoxidil Solutions in the Treatment of Female Pattern Hair Loss. Journal of the American Academy of Dermatology, 50, 541-553.

https://doi.org/10.1016/j.jaad.2003.06.014

[6] Price, V.H., Menefee, E. and Strauss, P.C. (1999) Changes in Hair Weight and Hair Count in Men with Aga, after Application of 5 Percent and 2 Percent Minoxidil. Journal of the American Academy of Dermatology, 41, 717-721. https://doi.org/10.1016/S0190-9622(99)70006-X

[7] Saifuddin, S., Ateeq, A., et al. (2015) A New Topical Formulation of Minoxidil and Finasteride Improves Hair Growth in Men with Androgenic Alopecia. Journal of Clinical \& Experimental Dermatology Research, 6, 1.

[8] Rinaldi, F., Pinto, D., Marzani, B., Rucco, M., Giuliani, G. and Sorbellini, E. (2018) Human Microbiome: What's New in Scalp Diseases. Journal of Translational Science, $4,1-4$.

[9] Tsukamoto, Y., Hendler, B. and Greenberg, S. (2018) Application of Ostrich Antibodies to the Restoration of Hair Growth, a Preliminary and Case Report. Journal of Cosmetics, Dermatological Sciences and Applications, 8, 179-184. https://doi.org/10.4236/jcdsa.2018.84019

[10] Schade, R., Pfister, C., Halatsch, R. and Henklein, P. (1991) Polyclonal IgY Antibodies from Chicken Egg Yolk. An Alternative to the Production of Mammalian IgG Type Antibodies in Rabbits. ATLA, 19, 403-419.

[11] Schade, R., Schniering, A. and Hlinak, A. (1992) Polyclonal Avian Antibodies Extracted from Egg Yolk as an Alternative to the Production of Antibodies in Mammals-A Review. ALTEX, 9, 43-56.

[12] Larsson, A., Balow, R.M., Lindahl, T.L. and Forsberg, P.O. (1993) Chicken Antibodies: Taking Advantage of Evolution: A Review. Poultry Science, 72, 1807-1812. https://doi.org/10.3382/ps.0721807

[13] Gross, M. and Speck, J. (1996) Avian Yolk Antibodies in Diagnosis and Research. Deutsche tierärztliche Wochenschrift, 103, 417-422.

[14] Leslie, G.A. and Clem, L.W. (1969) Phylogeny of Immunoglobulin Structure and Function. III. Immunoglobulins of the Chicken. The Journal of Experimental Medicine, 130, 1337-1352. https://doi.org/10.1084/jem.130.6.1337

[15] Thu, H.M., Myat, T.W., Win, M.M., Thant, K.Z., Rahman, S., Umeda, K., Nguyen, S.V., Icatlo, F.C., Higo-Moriguchi, K., Taniguchi, K., Tsuji, T., Oguma, K., Kim, S.J., Bae, H.S. and Choi, H.J. (2017) Chicken Egg Yolk Antibodies (IgY) for Prophylaxis and Treatment of Rotavirus Diarrhea in Human and Animal Neonates: A Concise Review. Korean Journal for Food Science of Animal Resources, 37, 1-9. https://doi.org/10.5851/kosfa.2017.37.1.1 
[16] Adachi, K., Handharvani, E., Sari, D.K., Takama, K., Fukuda, K., Endo, I., Yamamoto, R., Sawa, M., Tanaka, M., Konishi, I. and Tsukamoto, Y. (2008) Development of Neutralization Antibodies against Highly Pathogenic H5N1 Avian Influenza viRus Using Ostrich (Struthio camelus) Yolk. Molecular Medicine Reports, 1, 203-209.

[17] Adachi, K., Hagimori, K., Kikuta, T. and Tsukamoto, Y. (2008) Involvement of SC1, a Cell Adhesion Molecule, in the Progression of Mammary Gland Tumors. Molecular Medicine Reports, 1, 219-224. https://doi.org/10.3892/mmr.1.2.219

[18] Adachi, K., Kato, T., Kirimura, N., Kubota, Y., Shiba, H. and Tsukamoto, Y. (2014) Double Infections with Avian $\mathrm{A} / \mathrm{H} 5 \mathrm{~N} 1$ and Swine $\mathrm{A} / \mathrm{H} 1 \mathrm{~N} 1$ Influenza Viruses in Chickens. American International Journal of Biology, 2, 58-94. https://doi.org/10.15640/aijb.v2n3-4a6

[19] Kamiyama, Y., Adachi, K., Handharyani, E., Soejoedono, R.D., Kusano, T., Inai, M., Tsukamoto, M., Kashiwagi, S. and Tsukamoto, Y. (2011) Protection from Avian Influenza H5N1 Virus Infection with Antibody-Impregnated Filters. Virology Journal, 8, 54. https://doi.org/10.1186/1743-422X-8-54

[20] Tsukamoto, M., Hiroi, S., Adachi, K., Kato, H., Inai, M., Konishi, I., Tanaka, M., Yamamoto, R., Sawa, M., Handharvani, E. and Tsukamoto, Y. (2011) Antibodies against Swine Influenza Virus Neutralize the Pandemic Influenza Virus A H1N1. Molecular Medicine Reports, 4, 209-214. https://doi.org/10.3892/mmr.2011.410

[21] Tsukamoto, Y. (2012) Eggs of Ostrich, Save the Human Race: Antibody Discovery of Secret Dreams Atopy, Pandemic Influenza, HIV Also Shot Down.

[22] Tsukamoto, Y., Nishiura, K. and Maeda, O. (2013) Development of Functional Skin-Care Products Using Ostrich Antibodies. Fragance Journal, 6, 36-41.

[23] Tsukamoto, Y., Maeda, O., Shigekawa, G., Greenberg, S. and Hendler, B. (2018) Ostrich Antibody and Its Application to Skin Diseases, a Review and Case Report. Health, 10, 1357-1370. https://doi.org/10.4236/health.2018.1010105 